\title{
Developing an Agent-Based Model to Explore the Impact of Social Networks on Building Occupant Energy Consumption
}

\author{
Hui XIE* \\ School of Civil \& Resources Engineering \\ University of Science and Technology Beijing \\ Beijing, China \\ xiehui20000@sina.com \\ Ya-lin WANG \\ School of Civil \& Resources Engineering \\ University of Science and Technology Beijing \\ Beijing, China \\ 1029994096@qq.com
}

\author{
Tao-tao YIN \\ School of Civil \& Resources Engineering \\ University of Science and Technology Beijing \\ Beijing, China \\ taotaoyin@126.com
}

\begin{abstract}
Building occupant behavior has a great impact on the energy consumption in buildings. Indirect conservations are achieved through information transmission of energy efficiency measures from one occupant to his/her neighbours. Within the social network, energy use information can propagate through social networks and influence an occupant's energy consumption decisions. This paper develops a mathematical model for occupant energy consumption behavior and network information transmission. A case study of a residential building with one hundred and fifty occupants is used to illustrate the impact of occupants on the rest of their network in transferring information. Simulation results show that social interactions can improve energy efficiency and consequently reduce energy consumption of the whole building.
\end{abstract}

Keywords-agent-based modeling; building energy comsumption; social network; occupant behavior

\section{INTRODUCTION}

With concern growing about pollution, global climate change, and national security related to dependence on fossil fuel resources, much emphasis is being placed on reducing activities that contribute to the emission of greenhouse gases, other criteria pollutants and reducing our dependence on non-renewable fuels [1]. The building sector is one of the largest consumers of energy and accounts for $41.3 \%$ of consumption and $36 \%$ of related $\mathrm{CO}_{2}$ emissions [2]. In residential buildings, occupants generally have a high degree of control over their energy consumption through their actions (e.g. setting temperature cooling and heating points, light and equipment use). Previous research has shown that interactions among residents in a network increase energy savings which may be more cost effective than physical renovations of their buildings [3,4]. However, more comprehensive studies on the relationship between social networks and energy use are still needed. Therefore, the goal of this paper is to develop an agent-based model to emulate the energy consumption behavior of building occupants in social networks.

\section{BACKGROUND}

Energy conservation can be achieved through three aspects: (1) designing or retrofitting buildings to be more efficient, (2) enhancing building systems operation, and (3) occupants and their impact on building energy consumption [5-7]. Understanding that occupant behavior can substantially contribute to energy consumption in buildings, researchers have recently begun investigating how occupants interact and influence the energy use behavior of each other. For example, Göckeritz et al. proposed that the energy conservation behaviors of others have a strong positive correlation with an individual's conservation actions [8]. However, to date relatively little work has been done modeling the impact of social networks on building occupants' energy use behavior.

In the limited prior research, agent-based modeling $(\mathrm{ABM})$ has been used as a platform to model occupant behavior. $A B M$ is an analytical method that allows the modeling of heterogeneous agents in various types of environments with explicit decision rules [9]. These attributes make ABM particularly well-suited for modeling and understanding complex adaptive systems. In buildings, agents (i.e., occupants) are not homogeneous, are adaptive, and communicate through a complex system of social relationships.

\section{MODEL DEVELOPMENT}

The model consists of three submodels: agent norm, network generation, and building energy calculation and convergence. 


\section{A. Agent Norm}

Based on the information participants received in the pilot study, we constructed an algorithm for an individual to follow in simulation as the agent norm. This submodel determines how much each agent (occupant) changes their energy use during each time step and is based on established social influence network theory. The influence from other agents can be either positive, to use less energy or negative, to use more energy. According to the social influence network theory, in a social network with $N$ agents, for each time step, every agent calculates their new energy use (in $\mathrm{W} /$ person/day) taking into account the influence they received from the other agents of the social network using the following equation [10]:

$$
y_{i}^{(t+1)}=a_{i}\left(w_{i 1} y_{1}^{(t)}+w_{i 2} y_{2}^{(t)}+\cdots+w_{i N} y_{N}^{(t)}\right)+\left(1-a_{i}\right) y_{i}^{(1)}
$$

where $y_{i}^{(t+1)}$ is agent $i$ 's energy use at time $t+1, y_{i}^{(t)}$ is agent $i$ 's current energy use, $y_{i}^{(1)}$ is the initial energy use of agent $i$, $N$ is the number of agents (occupants) in the network, $w_{i j}$ is the weight of the influence of agent $j$ on agent $i\left(0 \leq w_{i j} \leq 1\right.$ and $\left.\sum_{j} w_{i j}=1\right)$, and $a_{i}$ is the susceptibility of agent $i$ to the influence of others $\left(0 \leq a_{i} \leq 1\right.$ and $\left.a_{i}=1-w_{i j}\right)$. Social influence network theory rests on a model of how individuals cognitively integrate conflicting opinions, but the outcome of this process depends on the social structure in which the process occurs.

The structural measure of an agent's susceptibility to influence is based on the agent's centrality in the communication network. Centrality is a well-established indicator of interpersonal influence, and the most elementary measure of centrality is the indegree of the agent for the measure of susceptibility:

$$
a_{i}=\left[1-\frac{1}{1+e^{-\left(d_{i}-2 \bar{d}\right)}}\right]^{1 / 2}
$$

where $d_{i}$ is the indegree of agent $i$, and $\bar{d}$ is the mean indegree of the agent.

The weight of interpersonal influence of one agent on another is calculated by considering the susceptibility of the agent and conditions that allow for likely interpersonal interaction, shared connections ( $i$ and $j$ both share ties with $k$ ) and reciprocity ( $i$ and $j$ share a connection) as given in (3):

$$
w_{i j}=\frac{S_{i} C_{i j}}{\sum_{k} C_{i k}}
$$

where $c_{i j}$ is the estimate of the probability of an interpersonal connection between agent $i$ and agent $j$ and $i \neq\{j, k\}$.

\section{B. Network Generation}

In this agent-based simulation, the environment in which occupants act is their social network. We assume that one agent's energy use can affect the energy use of other agents with varying degrees of probability. The quantity of consumption by neighbors of an agent's peer network may have a strong influence on modifying a given agent's energysaving behavior. On one hand, the agent' behaviors can actively change the energy consumption patterns in the whole network. On the other hand, once the environment changes, the feedback of changes will inversely affect agent's decision making processes.

There are four basic architectures for social networks: regular network, random network, small-world network, and scale-free network [11]. In this simulation, the random graph theory is used to generate complex networks (Fig. 1). Since 1950, large networks with no apparent design principles were described as random graph, which was proposed as the simplest and most straight forward realization of a complex network. A random network is a theoretical construct which contains links that are chosen completely at random with equal probability. The random network is highly disordered and low clustering, meanwhile its degree distribution is defined by Poisson distribution [11]. Some recent study indicate that large network, for example, World-Wide Web, Internet, metabolic and protein networks, have the scale-free property (the degree distribution follows a power law). However, since the residential network investigated in this study is relatively small compare to other networks, we only adopt random graph model without scale-free property.

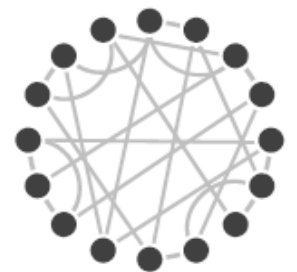

Figure 1. Graphical representation of a random network.

In this simulation, a building with $N$ occupants can be represented by $N$ nodes on a network. The interactions between the nodes are represented by edges on the network. The physical distance between each household is not considered in this paper. We assume that two nodes (occupants) are said to be connected when there is a mutual acknowledgement of friendship between them. In addition, we consider an undirected network where the path between two nodes is represented with $d_{\mathrm{i}, \mathrm{j}}=d_{\mathrm{j}, \mathrm{i}}$ and there is no self connection, that is $d_{\mathrm{i}, \mathrm{i}}=0$. The random network is created according to the method prescribed in Erdős and Rényi [12].

1) Building Energy Calculation and Convergence

The total building energy use (in W/day), $B E$, is calculated in this submodel using (4), which combines the updated energy uses of all building occupants as of the current time step: 


$$
B E=\sum_{i=1}^{N} y_{i}
$$

When the standard deviation of energy use between all agents falls below 1 watt and when the rate of change of the total building energy use mean and standard deviation over the last 50 time steps relative to the current time step has changed by less than 0.05 watts for each, the model is considered to have reached convergence. When convergence occurs, the simulation ends. Otherwise, the model moves to the next time step.

\section{SimUlation AND RESUlts}

In accordance with the objectives of this paper, a case study is conducted to verify the model behavior. A network of one hundred and fifty occupants is considered in this study.

Fig. 2 reflects a single simulation run of the dynamic energy consumption of twenty agents in the simulated network. Each agent can make decisions separately based on its own environment, although we assume that these decisions will affect decisions made by its neighbors in the peer network.

The percentages of the total expected energy use savings for twenty agents and the whole building are given in Fig. 3 , which shows the percentage savings after thirty days. The results show that agent 3 has the highest percentage of energy use savings after thirty days while the energy consumption of agent 15 increases very greatly. In addition, the energy use of the whole building reduces by $20.7 \%$.

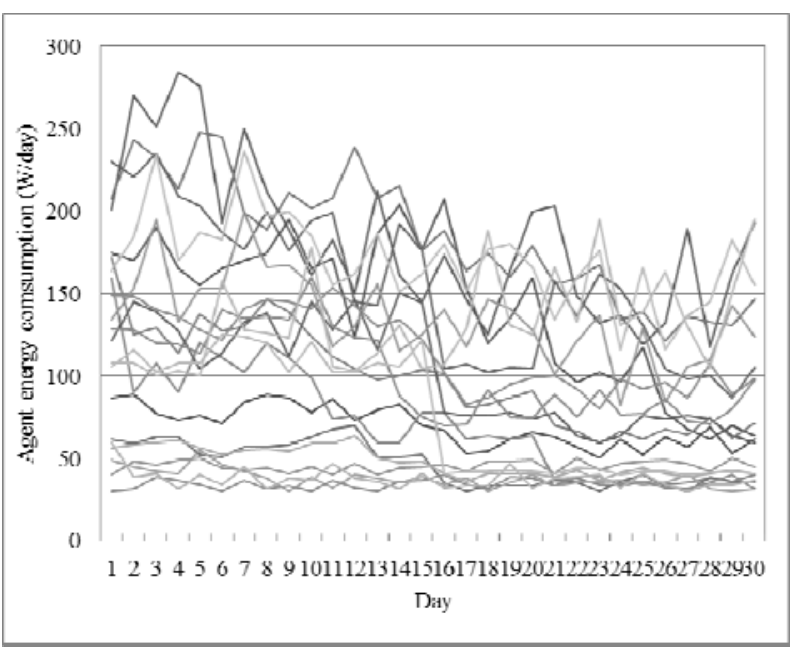

Figure 2. Agents' energy use performance.

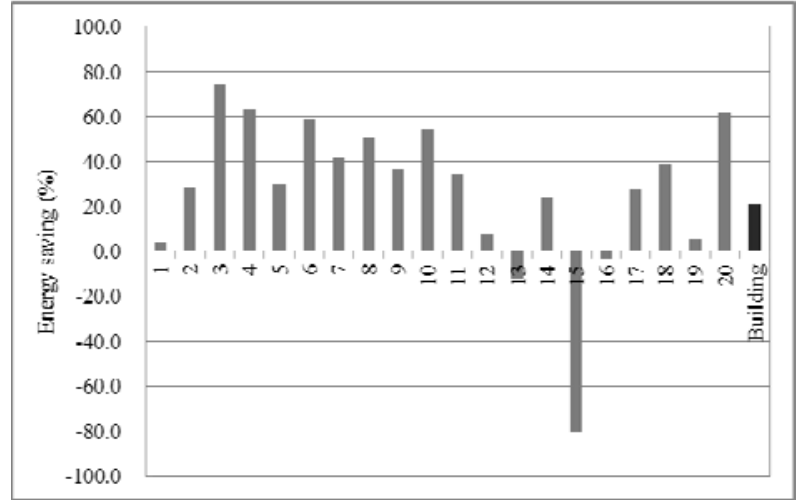

Figure 3. Energy use savings of agents and building.

The purpose of this simulation is to explore the effect of social network on occupants' energy saving behavior. In order to achieve this goal, we built an agent-based decision model to estimate such behaviors. However, this model needs further empirical validation from experimental data.

\section{CONCLUSIONS}

This paper presents an agent-based model that explores the relationship between social networks in buildings and energy use behaviors of building occupants. In existed document, energy conservation is seldom investigated at the social network level. Although experimental research recently has found that sharing energy use information through social networks promotes energy saving by building occupants [4, 13]. However, the characteristics of social networks vary from building to building, which means that the relationship between social network and energy saving behavior is difficult to generalize based solely on experimental data without the aid of simulations. Thus, a simulation model that can explain the residents' decision making process is a critical contribution to our understanding of how best to leverage the interpersonal relationships captured by peer networks to encourage energy savings.

In future research we plan to employ this model of an agent's simulated energy use to find some difference among different types of network structures. Some recent research shows that real social networks show scaling free and smallworld properties $[14,15]$. In addition, we will examine unique contributions of varying network properties on conservation behavior. The network size may be one condition that would lead to increasing or decreasing energy consumptions.

\section{REFERENCES}

[1] U.S. Congress, "Energy Independence and Security Act of 2007. 110t h Congress ed., H.R. 6," January 2007.

[2] US Energy Information Administration. Annual Energy Outlook. 201 0. http://www.eia.gov/forecasts/aeo/.

[3] X.Xu, J.E.Taylor, A.L.Pisello, P.J.Culligan, "The impact of place-bas ed affiliation network on energy conservation: an holistic model that $i$ ntegrates the influence of buildings, residents and the neighborhood c ontext, ”Energy and Buildings, vol. 55, pp. 637-646, 2012. 
[4] G. Peschiera, J. E. Taylor, and J. A. Siegel, "Responserelapse patterns of building occupant electricity consumption following exposure to $p$ ersonal, contextualized and occupant peer network utilization data," E nergy and Buildings, vol. 42, no. 8, pp. 1329-1336, 2010.

[5] Simpson, J., and McPherson, E. "Potential of tree shade for reducing $r$ esidential energy use in California." J. Arboric., vol. 22, no. 1, pp. 10 $-18,1996$.

[6] Hepbasli, A., and Akdemir, O. "Energy and exergy analysis of a grou nd source (geothermal) heat pump system." Energy Convers. Manage. vol. 45, no. 5, pp. 737-753, 2004.

[7] Foley, H. C. "Challenges and opportunities in engineered efficiency a nd habitability." AIChE J., vol. 58, no. 3, pp. 658-667, 2012.

[8] Göckeritz, S., P. W. Schultz, T. Rendón, R. Cialdini, N. J. Goldstein a nd V. Griskevicius. "Descriptive normative beliefs and conservation b ehavior: the moderating roles of personal involvement and injunctive normative beliefs." European Journal of Social Psychology, vol. 40, p p. 514-523, 2010 .
[9] Gilbert, N. Agent-based models, 7th Ed., Sage, Los Angeles. 2008.

[10] N. E. Friedkin, "Norm formation in social influence networks," Social Networks, vol. 23, no. 3, pp. 167-189, 2001.

[11] Graham, W. The Art, Soul, Science of Beholding Nature - Patterns In Nature. 2013. Retrieved from Patterns In Nature: http://www.patternsi nnature.org/PinTextBook.html

[12] Erdös, P. and A. Rényi. "On the evolution of random graphs." Publ. Math. Inst. Hung. Acad. Sci, vol. 5, pp. 17-61, 1960.

[13] Petersen, J., V. Shunturov, K. Janda, G. Platt, and K. Weinberger. "D ormitory residents reduce electricity consumption when exposed to re al-time visual feedback and incentives." International Journal of Susta inability in Higher Education, vol. 8, no. 1, pp. 16-33, 2007.

[14] Barabási, A. and R. Albert. "Emergence of Scaling in Random Netwo rks" Science, vol. 286, no. 5439, pp. 509-512, 1999.

[15] Watts, D. and S. Strogatz. "Collective dynamics of small-world netw orks." Nature, vol. 393, pp. 440-442, 1998. 